\title{
Necessary conditions for double summability factor theorem
}

\author{
Ekrem Savaş
}




\title{
NECESSARY CONDITIONS FOR DOUBLE SUMMABILITY FACTOR THEOREM
}

\author{
EKREM SAVAŞ
}

Received 20 December, 2009

\begin{abstract}
We obtain necessary conditions for the series $\sum \sum c_{m n}$, which is absolutely summable of order $k$ by a doubly triangular matrix method $A$, to be such that $\sum \sum c_{m n} \lambda_{m n}$ is absolutely summable of order $k$ by a doubly triangular matrix $B$.
\end{abstract}

2000 Mathematics Subject Classification: 40F05, 40D25

Keywords: absolute summability factors, doubly triangular summability

\section{INTRODUCTION}

A doubly infinite matrix $A=\left(a_{m n i j}\right)$ is said to be doubly triangular if $a_{m n i j}=0$ for $i>m$ and $j>n$. The mn-th terms of the $A$-transform of a double sequence $\left\{s_{m n}\right\}$ is defined by

$$
T_{m n}=\sum_{i=0}^{m} \sum_{j=0}^{n} a_{m n i j} s_{i j} .
$$

A series $\sum \sum c_{m n}$, with partial sums $s_{m n}$ is said to be absolutely $A$-summable, of order $k \geq 1$, if

$$
\sum_{m=1}^{\infty} \sum_{n=1}^{\infty}(m n)^{k-1}\left|\Delta_{11} T_{m-1, n-1}\right|^{k}<\infty,
$$

where for any double sequence $\left\{u_{m n}\right\}$, and for any four-fold sequence $\left\{a_{m n i j}\right\}$, we define

$$
\begin{aligned}
\Delta_{11} u_{m n} & =u_{m n}-u_{m+1, n}-u_{m, n+1}+u_{m+1, n+1}, \\
\Delta_{11} a_{m n i j} & =a_{m n i j}-a_{m+1, n, i, j}-a_{m, n+1, i, j}+a_{m+1, n+1, i, j}, \\
\Delta_{i j} a_{m n i j} & =a_{m n i j}-a_{m, n, i+1, j}-a_{m, n, i, j+1}+a_{m, n, i+1, j+1}, \\
\Delta_{i 0} a_{m n i j} & =a_{m n i j}-a_{m, n, i+1, j}, \quad \text { and } \\
\Delta_{0 j} a_{m n i j} & =a_{m n i j}-a_{m, n, i, j+1} .
\end{aligned}
$$

The one-dimensional version of (1.1) appears in [1]. 
Associated with $A$ there are two matrices $\bar{A}$ and $\hat{A}$ defined by

$$
\bar{a}_{m n i j}=\sum_{\mu=i}^{m} \sum_{\nu=j}^{n} a_{m n \mu \nu}, 0 \leq i \leq m, 0 \leq j \leq n, m, n=0,1, \ldots,
$$

and

$$
\hat{a}_{m n i j}=\Delta_{11} \bar{a}_{m-1, n-1, i, j}, 0 \leq i \leq m, 0 \leq j \leq n, m, n=1,2, \ldots .
$$

It is easily verified that $\hat{a}_{0000}=\bar{a}_{0000}=a_{0000}$. In [3] it is shown that

$$
\hat{a}_{m n i j}=\sum_{\mu=0}^{i-1} \sum_{\nu=0}^{j-1} \Delta_{11} a_{m-1, n-1, \mu, \nu} .
$$

Thus $\hat{a}_{m n i 0}=\hat{a}_{m n 0 j}=0$.

Let $x_{m n}$ denote the mn-th term of the $A$-transform of the sequence of partial sums $\left\{s_{m n}\right\}$ of the series $\sum \sum c_{m n}$.

Then

$$
\begin{aligned}
x_{m n} & =\sum_{i=0}^{m} \sum_{j=0}^{n} a_{m n i j} s_{i j}=\sum_{i=0}^{m} \sum_{j=0}^{n} a_{m n i j} \sum_{\mu=0}^{i} \sum_{\nu=0}^{j} c_{\mu \nu} \lambda_{\mu \nu} \\
& =\sum_{\mu=0}^{m} \sum_{\nu=0}^{n} \sum_{i=\mu}^{m} \sum_{j=v}^{n} a_{m n u v} c_{\mu \nu} \lambda_{\mu \nu} \\
& =\sum_{\mu=0}^{m} \sum_{\nu=0}^{n} \bar{a}_{m n \mu \nu} c_{\mu \nu} \lambda_{\mu \nu},
\end{aligned}
$$

and a direct calculation verifies that

$$
X_{m n}:=\Delta_{11} x_{m-1, n-1}=\sum_{\nu=1}^{m} \sum_{\mu=1}^{n} \hat{a}_{m n \mu \nu} c_{\mu \nu} \lambda_{\mu \nu},
$$

since

$$
\bar{a}_{m-1, n-1, m, v}=a_{m-1, n-1, \mu, n}=\hat{a}_{m, n-1, \mu, n}=\hat{a}_{m-1, n, m, n}=0 .
$$

In a recent paper Savas and Rhoades[2] obtained sufficient conditions for the series $\sum \sum c_{m n}$, which is absolutely summable of order $k$ by a doubly triangular matrix method $A$, to be such that $\sum \sum c_{m n} \lambda_{m n}$ is absolutely summable of order $k$ by a doubly triangular matrix $B$.

In this paper we obtain necessary conditions for the series $\sum \sum c_{m n}$, which is absolutely summable of order $k$ by a doubly triangular matrix method $A$, to be such that $\sum \sum c_{m n} \lambda_{m n}$ is absolutely summable of order $k$ by a doubly triangular matrix $B$. 


\section{MAIN THEOREM}

Theorem 1. Let $A$ and $B$ be doubly triangular matrices with $A$ satisfying

$$
\sum_{m=u+1}^{\infty} \sum_{n=v+1}^{\infty}(m n)^{k-1}\left|\Delta_{u v} \hat{a}_{m n u v}\right|^{k}=O\left(M^{k}\left(\hat{a}_{u v u v}\right)\right),
$$

where

$$
M\left(\hat{a}_{u v u v}\right):=\max \left\{\left|\hat{a}_{u v u v}\right|,\left|\Delta_{u 0} \hat{a}_{u+1 v, u, v}\right|,\left|\Delta_{0 v} \hat{a}_{u v+1, u, v}\right|\right\} .
$$

Then the necessary conditions of the fact that the $|A|_{k}$ summability of $\sum \sum c_{m n}$ implies the $|B|_{k}$ summability of $\sum \sum c_{m n} \lambda_{m n}$ are the following items:

(i) $\left|\hat{b}_{u v u v} \lambda_{u v}\right|=O\left(M\left(\hat{a}_{u v u v}\right)\right)$,

(ii) $\left|\Delta_{u 0} \hat{b}_{u+1, v, u, v} \lambda_{u v}\right|=O\left(M\left(\hat{a}_{u v u v}\right)\right)$,

(iii) $\left|\Delta_{0 v} \hat{b}_{u, v+1, u, v} \lambda_{u v}\right|=O\left(M\left(\hat{a}_{u v u v}\right)\right)$,

(iv) $\sum_{m=u+1}^{\infty} \sum_{n=v+1}^{\infty}(m n)^{k-1}\left|\Delta_{u v} \hat{b}_{m n u v} \lambda_{u v}\right|^{k}=O\left((u v)^{k-1} M^{k}\left(\hat{a}_{u v u v}\right)\right)$,

(v) $\sum_{m=u+1}^{\infty} \sum_{n=v+1}^{\infty}(m n)^{k-1}\left|\hat{b}_{m, n, u+1, v+1} \lambda_{u+1, v+1}\right|^{k}=$ $O\left(\sum_{m=u+1}^{\infty} \sum_{n=v+1}^{\infty}(m n)^{k-1}\left|\hat{a}_{m, n, u+1, v+1}\right|^{k}\right)$.

Proof. For $k \geq 1$ define

$$
\sum_{m=1}^{\infty} \sum_{n=1}^{\infty}(m n)^{k-1}\left|Y_{m n}\right|^{k}<\infty
$$

whenever

$$
\sum_{m=1}^{\infty} \sum_{n=1}^{\infty}(m n)^{k-1}\left|X_{m n}\right|^{k}<\infty
$$

where

$$
\begin{gathered}
Y_{m n}=\Delta_{11} y_{m-1, n-1}, \\
y_{m n}=\sum_{i=0}^{m} \sum_{j=0}^{n} \bar{b}_{m n i j} c_{i j} \lambda_{i j} .
\end{gathered}
$$

The space of sequences satisfying (2.3) is a Banach space if it is normed by

$$
\|X\|=\left(\left|X_{00}\right|^{k}+\left|X_{01}\right|^{k}+\left|X_{10}\right|^{k}+\sum_{m=1}^{\infty} \sum_{n=1}^{\infty}(m n)^{k-1}\left|X_{m n}\right|^{k}\right)^{1 / k} .
$$


We shall also consider the space of sequences $\left\{Y_{m n}\right\}$ that satisfy (2.2). This space is also a BK-space with respect to the norm

$$
\|Y\|=\left(\left|Y_{00}\right|^{k}+\left|Y_{01}\right|^{k}+\left|Y_{10}\right|^{k}+\sum_{m=1}^{\infty} \sum_{n=1}^{\infty}(m n)^{k-1}\left|Y_{m n}\right|^{k}\right)^{1 / k}
$$

The transformation $\sum_{i=0}^{m} \sum_{j=0}^{n} \bar{b}_{m n i j} c_{i j} \lambda_{i j}$ maps sequences satisfying (2.3) into sequences satisfying (2.2). By the Banach-Steinhaus Theorem there exists a constant $K>0$ such that

$$
\|Y\| \leq K\|X\| .
$$

For fixed $u, v$, the sequence $\left\{c_{i j}\right\}$ is defined by $c_{u v}=c_{u+1, v+1}=1, c_{u+1, v}=c_{u, v+1}=$ $-1, c_{i j}=0$, otherwise,

$$
X_{m n}=\left\{\begin{array}{l}
0, \quad m \leq u, n<v \\
0, \quad m<u, n \leq v \\
\hat{a}_{m n u v}, m=u, n=v \\
\Delta_{u 0} \hat{a}_{m n u v}, m=u+1, n=v, \\
\Delta_{0 v} \hat{a}_{m n u v}, m=u, n=v+1, \\
\Delta_{u v} \hat{a}_{m n u v}, m>u, n>v
\end{array}\right.
$$

and

$$
Y_{m n}=\left\{\begin{array}{l}
0, \quad m \leq u, n<v \\
0, \quad m<u, n \leq v \\
\hat{b}_{m n u v} \lambda_{u v}, m=u, n=v \\
\Delta_{u 0} \hat{b}_{m n u v} \lambda_{u v}, m=u+1, n=v \\
\Delta_{0 v} \hat{b}_{m n u v} \lambda_{u v}, m=u, n=v+1 \\
\Delta_{u v} \hat{b}_{m n u v} \lambda_{u v}, m>u, n>v
\end{array}\right.
$$

From (2.4) and (2.5) it follows that

$$
\begin{aligned}
\|X\|= & \left\{(u v)^{k-1}\left|\hat{a}_{u v u v}\right|^{k}+((u+1) v)^{k-1}\left|\Delta_{u 0} a_{u+1, v, u, v}\right|^{k}\right. \\
& +(u(v+1))^{k-1}\left|\Delta_{0 v} a_{u, v+1 u, v}\right|^{k} \\
& \left.+\sum_{m=u+1}^{\infty} \sum_{n=v+1}^{\infty}(m n)^{k-1}\left|\Delta_{u v} \hat{a}_{m n u v}\right|^{k}\right\}^{1 / k}
\end{aligned}
$$

and

$$
\begin{aligned}
\|Y\|= & \left\{\left.(u v)^{k-1} \hat{b}_{u v u v} \lambda_{u v}\right|^{k}+((u+1) v)^{k-1}\left|\Delta_{u 0} \hat{b}_{u+1, v, u, v} \lambda_{u v}\right|^{k}\right. \\
& +(u(v+1))^{k-1}\left|\Delta_{0 v} \hat{b}_{u, v+1 u, v} \lambda_{u v}\right|^{k}
\end{aligned}
$$




$$
\left.+\sum_{m=u+1}^{\infty} \sum_{n=v+1}^{\infty}(m n)^{k-1}\left|\Delta_{u v} \hat{a}_{m n u v} \lambda_{u v}\right|^{k}\right\}^{1 / k} .
$$

Substituting (2.7) and (2.8) into (2.6), along with (2.1), gives

$$
\begin{gathered}
(u v)^{k-1}\left|\hat{b}_{u v u v} \lambda_{u v}\right|^{k}+((u+1) v)^{k-1}\left|\Delta_{u 0} \hat{b}_{u+1 v, u, v} \lambda_{u v}\right|^{k} \\
+(u(v+1))^{k-1}\left|\Delta_{0 v} \hat{b}_{u, v+1 u, v} \lambda_{u v}\right|^{k} \\
+\sum_{m=u+1}^{\infty} \sum_{n=v+1}^{\infty}(m n)^{k-1}\left|\Delta_{u v} \hat{b}_{m n u v} \lambda_{u v}\right|^{k} \leq K^{k}\left\{(u v)^{k-1}\left|\hat{a}_{u v u v}\right|^{k}\right. \\
((u+1) v)^{k-1}\left|\Delta_{u 0} \hat{a}_{u+1 v, u, v}\right|^{k}+\left.(u(v+1))^{k-1} \Delta_{u 0} \hat{a}_{u, v+1 u, v}\right|^{k} \\
\left.+\sum_{m=u+1}^{\infty} \sum_{n=v+1}^{\infty}(m n)^{k-1}\left|\Delta_{u v} \hat{a}_{m n u v}\right|^{k}\right\} \\
=K^{k}\left\{O(1)(u v)^{k-1} M^{k}\left(\hat{a}_{u v u v}\right)\right\} .
\end{gathered}
$$

The above inequality implies that each term of the left hand side is $O\left(\left\{(u v)^{k-1} M^{k}\left(\hat{a}_{u v u v}\right)\right\}\right)$.

Using the first term one obtains

$$
(u v)^{k-1}\left|\hat{b}_{u v u v} \lambda_{u v}\right|^{k}=O\left(\left\{(u v)^{k-1} M^{k}\left(\hat{a}_{u v u v}\right)\right\}\right) .
$$

Thus

$$
\left|\hat{b}_{u v u v} \lambda_{u v}\right|=O\left(M\left(\hat{a}_{u v u v}\right)\right)
$$

which is condition (i).

In a similar manner one obtains conditions (ii) - (iv).

Using the sequence, defined by $c_{u+1, v+1}=1$, and $c_{i j}=0$ otherwise, yields

$$
X_{m n}=\left\{\begin{array}{l}
0, \quad m \leq u+1, n \leq v, \\
0, \quad m \leq u, n \leq v+1, \\
\hat{a}_{m, n, u+1, v+1}, m \geq u+1, n \geq v+1
\end{array}\right.
$$

and

$$
Y_{m n}=\left\{\begin{array}{l}
0 . \quad m \leq u+1, n \leq v, \\
0, \quad m \leq u, n \leq v+1, \\
\hat{b}_{m, n, u+1, v+1} \lambda_{u+1, v+1}, m \geq u+1, n \geq v+1 .
\end{array}\right.
$$

The corresponding norms are

$$
\|X\|=\left\{\sum_{m=u+1}^{\infty} \sum_{n=v+1}^{\infty}(m n)^{k-1}\left|\hat{a}_{m, n, u+1, v+1}\right|^{k}\right\}^{1 / k}
$$


and

$$
\|Y\|=\left\{\sum_{m=u+1}^{\infty} \sum_{n=v+1}^{\infty}(m n)^{k-1}\left|\hat{b}_{m, n, u+1, v+1} \lambda_{u+1, v+1}\right|^{k}\right\}^{1 / k} .
$$

Applying (2.6), we have

$$
\begin{aligned}
& \sum_{m=u+1}^{\infty} \sum_{n=v+1}^{\infty}(m n)^{k-1}\left|\hat{b}_{m, n, u+1, v+1} \lambda_{u+1, v+1}\right|^{k} \\
\leq & K^{k}\left\{\sum_{m=u+1}^{\infty} \sum_{n=v+1}^{\infty}(m n)^{k-1}\left|\hat{a}_{m, n, u+1, v+1}\right|^{k}\right\},
\end{aligned}
$$

which implies (v).

Every summability factor theorem becomes an inclusion theorem by setting each $\lambda_{m n}=1$.

Corollary 1. Let $A$ and $B$ two doubly triangular matrices, A satisfying (2.1). Then necessary conditions of the fact that the $|A|_{k}$ summability of $\sum \sum c_{m n}$ implies the $|B|_{k}$ summability of $\sum \sum c_{m n}$ are the following items:

(i) $\left|\hat{b}_{u v u v}\right|=O\left(M\left(\hat{a}_{u v u v}\right)\right)$,

(ii) $\left|\Delta_{u 0} \hat{b}_{u+1, v, u, v}\right|=O\left(M\left(\hat{a}_{u v u v}\right)\right)$,

(iii) $\left|\Delta_{0 v} \hat{b}_{u, v+1, u, v}\right|=O\left(M\left(\hat{a}_{u v u v}\right)\right)$,

(iv) $\sum_{m=u+1}^{\infty} \sum_{n=v+1}^{\infty}(m n)^{k-1}\left|\Delta_{u v} \hat{b}_{m n u v}\right|^{k}=O\left((u v)^{k-1} M^{k}\left(\hat{a}_{u v u v}\right)\right)$, and

(v) $\sum_{m=u+1}^{\infty} \sum_{n=v+1}^{\infty}(m n)^{k-1}\left|\hat{b}_{m, n, u+1, v+1}\right|^{k}$

$=O\left(\left\{\sum_{m=u+1}^{\infty} \sum_{n=v+1}^{\infty}(m n)^{k-1}\left|\hat{a}_{m, n, u+1, v+1}\right|^{k}\right\}^{1 / k}\right)$.

Proof. To prove Corollary 1 simply put $\lambda_{m n}=1$ in Theorem 1 .

We shall call a doubly infinite matrix a product matrix if it can be written as the termwise product of two singly infinite matrices $F$ and $G$; i.e., $a_{m n i j}=f_{m i} g_{n j}$ for each $i, j, m, n$.

A doubly infinite weighted mean matrix $P$ has nonzero entries $p_{i j} / P_{m n}$, where $p_{00}$ is positive and all of the other $p_{i j}$ are nonnegative, and $P_{m n}:=\sum_{i=0}^{m} \sum_{j=0}^{n} p_{i j}$. If $P$ is a product matrix then the nonzero entries are $p_{i} q_{j} / P_{m} Q_{n}$, where $p_{0}>0, p_{i}>$ 0 for $i>0, q_{0}>0, q_{i} \geq 0$ for $j>0$ and $P_{m}:=\sum_{i=0}^{m} p_{i}, Q_{n}:=\sum_{j=0}^{n} q_{j}$. Now we have the following corollary 
Corollary 2. Let $B$ be a doubly triangular matrix, $P$ a product weighted mean matrix satisfying

$$
\sum_{m=u+1}^{\infty} \sum_{n=v+1}^{\infty}(m n)^{k-1}\left|\Delta_{u v}\left(\frac{p_{m} q_{n} P_{u-1} Q_{v-1}}{P_{m} P_{m-1} Q_{n} Q_{n-1}}\right)\right|^{k}=O\left(\frac{p_{u} q_{v}}{P_{u} Q_{v}}\right) .
$$

Then necessary conditions for $\sum \sum c_{m n}$ summable $|P|_{k}$ to imply that $\sum \sum c_{m n} \lambda_{m n}$ is summable $|B|_{k}$ are

(i) $\left|\hat{b}_{u v u v} \lambda_{u v}\right|=O\left(\frac{p_{u} q_{v}}{P_{u} Q_{v}}\right)$,

(ii) $\left|\Delta_{u 0} \hat{b}_{u+1, v, u, v} \lambda_{u v}\right|=O\left(\frac{p_{u} q_{v}}{P_{u} Q_{v}}\right)$,

(iii) $\left|\Delta_{0 v} \hat{b}_{u, v+1, u, v} \lambda_{u v}\right|=O\left(\frac{p_{u} q_{v}}{P_{u} Q_{v}}\right)$,

(iv) $\sum_{m=u+1}^{\infty} \sum_{n=v+1}^{\infty}(m n)^{k-1}\left|\Delta_{u v} \hat{b}_{u v u v} \lambda_{u v}\right|^{k}=O\left((u v)^{k-1}\left(\frac{p_{u} q_{v}}{P u Q_{v}}\right)^{k}\right)$, and

(v) $\sum_{m=u+1}^{\infty} \sum_{n=v+1}^{\infty}(m n)^{k-1}\left|\hat{b}_{m, n, u+1, v+1} \lambda_{u+1, v+1}\right|^{k}=O(1)$.

\section{ACKNOWLEDGEMENTS}

I wish to thank the referee for his careful reading of the manuscript and for his helpful suggestions.

\section{REFERENCES}

[1] B. E. Rhoades and E. Savaş, "General summability factor theorems and applications," Sarajevo J. Math., vol. 1(13), no. 1, pp. 59-73, 2005.

[2] E. Savaş and B. E. Rhoades, "Double summability factor theorems and applications," Math. Inequal. Appl., vol. 10, no. 1, pp. 125-149, 2007.

[3] E. Savaş and B. E. Rhoades, "Double absolute summability factor theorems and applications," Nonlinear Anal., vol. 69, no. 1, pp. 189-200, 2008.

Author's address

\section{Ekrem Savaş}

Current address: Istanbul Ticaret University Department of Mathematics, Selman-i Pak Cad. 34672, Üsküdar-Istanbul/Turkey

E-mail address: ekremsavas@yahoo.com 